\title{
PERIPROSTATIC HAEMATOMA AND PROSTATIC ABSCESS IN THE NEONATAL PERIOD
}

BY

\author{
D. INNES WILLIAMS and ANTONIO G. MARTINS \\ From The Hospital for Sick Children, Great Ormond Street, London
}

(RECEIVED FOR PUBLICATION MAY 25, 1959)

The object of this contribution is to draw attention to a hitherto undescribed lesion, peri-prostatic haematoma, and to discuss its relationship to prostatic abscess occurring in the neonatal period. Both disorders have been wholly or partially responsible for a severe but remediable lower urinary tract obstruction in a series of infants observed at The Hospital for Sick Children, Great Ormond Street. The aetiology of the conditions, and even the exact pathological anatomy, remain obscure for, since all cases survived, only clinical data concerning them are available.

Prostatic abscess in an infant has been described on a number of occasions (Campbell, 1929; Szenkier, 1929; Fox, 1934; Alison and Le Tan Vinh, 1952; Boy, Wallon and Lauras, 1953). We have also treated a staphylococcal prostatic abscess in an infant, a case previously reported by George (1953), which is now summarized below.

Case 1. A previously healthy boy, aged 1 month, developed a hard red swelling on both sides of the scrotum; four days later he vomited after feeds and his abdomen was distended, due mainly to an enlarged bladder. Catheterization brought a marked but not complete improvement. The following day gross distension of the abdomen recurred and again catheterization released a clear urine. An abscess pointed in the right scrotum and was opened, releasing $7 \mathrm{ml}$. of thick sticky pus, which on culture grew Staphylococcus aureus. Subsequently catheterization was unsuccessful and the boy was transferred to The Hospital for Sick Children. His temperature had been only slightly raised and he was having small frequent green stools with much mucus. On admission the abdomen was distended and rather tympanitic and rectal examination revealed a small rectum compressed anteriorly by a cystic swelling, apparently in continuity with the bladder. The left epididymis was thickened; the right testicle and scrotum were hard with a discharging sinus inferiorly. Enlarged lymph nodes were present in both groins. The blood urea was normal and the urine sterile, but there was a leucocytosis (W.B.C. $21,200,73 \%$ neutrophils). On the same day a suprapubic cystostomy was performed and a tensely distended, thin-walled bladder was found. A fluctuant swelling was present beneath the trigone, elongating it and extending downwards behind the urethra. The bladder itself was normal. A perineal incision was made immediately in front of the rectum and a sinus forceps was guided into a large abscess cavity by a finger in the rectum. About 2 oz. of thick pus were evacuated. On culture there was a heavy growth of coagulase-positive staphylococci and a few colonies of Escherichia coli.

After three days there was a recurrence of the scrotal abscess and a certain amount of greenish-yellow pus was obtained, which on culture again grew Staph. aureus. Rectal examination showed some thickening in the prostatic region when the child was discharged on the fifteenth day. Two months later he was completely symptom-free.

There can be little doubt that the abscess in this case arose within the prostatic gland. It was a strictly midline swelling, closely resembling a prostatic tumour, and the simultaneous occurrence of epididymitis confirmed its prostatic origin. As in similar cases occurring in adult life, there must be some doubt as to whether the infection, which was clearly haematogenous, started in the prostate or in the epididymis, and the primary focus outside the genital tract was not identified. The presence of a staphylococcus suggests a skin sepsis, but none was found and the umbilicus was clean.

The cases which we place in the category of periprostatic haematoma (Cases 2 and 4, which are reported in full later) present several points of distinction. A mass was present in the pelvis which contained only old blood, sterile in Case 2, and infected with Esch. coli in Case 4 at a time when a blood culture also grew Esch. coli. The swelling as felt per rectum was lateral rather than median and was broadly based on the lateral pelvic wall, bulging into the cavity of the pelvis from one side only. It was thus distinguishable from a mass arising within the pelvic viscera. It appeared, therefore, that this effusion of blood had occurred in the fascial spaces 
alongside the prostate and bladder a little in front of the rectum. No cause of the effusion could be identified, none of these cases had bleeding from any other source and there did not appear to be any coagulation defect. One infant had Esch. coli septicaemia in association with a urinary infection and in addition a urethral obstruction due to congenital valves. The possibility of a descending arteritis in the umbilical artery was considered as a source of the haemorrhage but there was no evidence of umbilical sepsis to support this hypothesis.

The clinical findings in Case 3 resembled those in Cases 2 and 4 but the swelling in the pelvis contained pus rather than blood: its lateral position suggested that suppuration might have occurred in a periprostatic haematoma but it could equally have arisen as a metatastic abscess in the prostate. In Case 5 the swelling resolved without surgical intervention; it is possible that an abscess cavity burst into the urethra in this child, suggesting a true prostatic lesion.

Retention of urine was the dominant clinical feature in all these infants. It was abrupt in onset and, although occurring within the first month of life, in only one was there any suggestion of a congenital abnormality. The bladder was tensely distended, the kidneys palpable and the blood urea raised. The urinary obstruction was due to pressure on the urethra and bladder neck: it was relieved as soon as the abscess or haematoma was evacuated. Urinary infection was present at some time in all instances, but probably resulted from catheterization. In Case 4 Esch. coli was already present before instrumentation and a septicaemia with severe collapse and dehydration at first concealed the real nature of the condition.

Drainage was obtained through a perineal incision: this method entails least disturbance of other organs but the track is apt to be long and narrow. It was found that refilling of the cavity could be minimized by stripping down the area with a finger in the rectum, and the fourth case settled down within a few days on this routine. Convalescence in all cases has been prolonged and the urinary infection has been difficult to eliminate. Some permanent damage to the upper urinary tract must be anticipated although the blood urea in all cases returned to normal.

\section{Case Reports}

Case 2. This boy, a first child, was born at full term on June 18, 1955 in a normal delivery and weighed $8 \mathrm{lb} .12 \mathrm{oz}$. The mother's pregnancy and puerperal period were uneventful apart from moderate anaemia. He was breast fed for one week, and then thrived on bottle feeding (N.D. milk) having no complaints until 4 weeks old when, on July 13, he started vomiting after feeds and having diarrhoea with loose greenish stools without mucus or blood. The parents also noticed that the child cried after passing urine, although he had a good stream with no dribbling. On account of worsening of his symptoms, he was admitted to Thurrock Hospital on July 15 and was found to have abdominal distension and a full bladder; catheterization produced $13 \mathrm{oz}$. of pale clear urine. When catheterized again that evening another $14 \mathrm{oz}$. were obtained. On July 16 the bladder was again distended up to the umbilicus and the infant's general condition was poor. Catheterization was then unsuccessful: a suprapubic lumbar puncture needle was inserted and $9 \mathrm{oz}$. of urine were removed.

The child was transferred to The Hospital for Sick Children and on admission on July 16 he did not look well but cried fairly lustily. His colour was fair but he was moderately dehydrated, weighing $9 \mathrm{lb} .4 \frac{1}{2} \mathrm{oz}$. There was moderate tachypnoea and a suggestion of meningism but no pyrexia. The abdomen was distended with subcutaneous veins rather more obvious than usual. The bladder was up to the umbilicus but the kidneys could not be felt. Rectal examination revealed a large anterior mass bulging into the rectum. The provisional diagnosis was congenital urethral obstruction. Investigations showed:

(16.7.55) blood urea $134 \mathrm{mg} . / 100 \mathrm{ml}$. ; serum electrolytes: $\mathrm{Cl} 713 \mathrm{mg} . / 100 \mathrm{ml} .(121 \mathrm{mEq} . / 1$.), Na $345 \mathrm{mg} . / 100 \mathrm{ml}$. (150 mEq./1.), K $21 \mathrm{mg} . / 100 \mathrm{ml}$. (5.4 mEq./1.), $\mathrm{CO}_{2}$ 21.2 vol. \% (9.5 mEq./1.); $\mathrm{Hb} 86 \%$ or $12.73 \mathrm{~g}$. $/ 100 \mathrm{ml}$. (17.7.55.)blood urea $142 \mathrm{mg} . / 100 \mathrm{ml}$; urine pale, turbid; S.G. 1016; protein $160 \mathrm{mg} . \%$; deposit: masses of W.B.C.s in uncentrifuged urine; heavy growth of Proteus on culture.

(18.7.55.) bleeding time 4 min. 55 sec.; clotting time 1 min. 40 sec.

Dehydration was corrected with intravenous fluids and bilateral pyelostomy was performed for drainage. The following day there was still a palpable cystic mass in the prostatic region and an operation was performed under a general anaesthetic. After catheterization, which obtained $5 \mathrm{oz}$. of urine, an exploratory needle was introduced through the perineum and entered a cystic cavity from which some dark blood was withdrawn. A small perineal incision was then made and through it $1 \mathrm{oz}$. of similar material was obtained. This cyst appeared to have no connexion with either the rectum or the lower urinary tract. A rubber drainage tube was left in place in the wound and the urethral catheter was left to drain the bladder. The contents of the cavity showed numerous R.B.C.s; an occasional polymorph but no organisms were seen. On culture there was no growth.

During the following days his general condition improved steadily and his blood urea fell. The left nephrostomy tube was removed on July 18, the right nephrostomy tube on July 22 and the urethral catheter on July 23. At that time the urine was sterile. As there seemed to be inadequate drainage and he was not able to empty his bladder completely, the wound was reexplored on July 26 and another drainage tube was 
inserted. A sinogram was performed and outlined the cavity which was unconnected with any of the pelvic organs (Fig. 1). A wound swab showed on culture a light growth of coagulase-positive staphylococci and Esch. coli. In the following days the discharge became frankly purulent but diminished progressively in amount, and the pelvic mass gradually subsided. On September 4 he developed gastro-enteritis and he required several intravenous infusions and antibiotic treatment before he finally settled down. When he was discharged on October 2 he was having no difficulty with micturition, and the pelvic mass had practically disappeared, leaving only a rather general induration which extended across both sides of the pelvis.

When re-investigated three and a half years later, the urinary tract was normal on intravenous pyelography and cystoscopy, although he was still enuretic.

Case 3. A premature first-born male child was born on September 17, 1957, by normal delivery. He weighed $4 \mathrm{lb}$. $14 \mathrm{oz}$. and looked normal. He thrived on bottle feeding (N.D. milk) and had no complaints until 5 weeks old when, on October 23, he ceased to pass urine. His legs and lower abdomen were moderately oedematous and he vomited after all feeds. On the same day he was admitted to the Whittington Hospital where he was found to have a distended bladder.

He was transferred to The Hospital for Sick Children on October 24, when he looked ill and rather pale and greyish in colour but hydration was fair. He weighed $7 \mathrm{lb} .5 \frac{1}{2} \mathrm{oz}$. There was moderate oedema in the legs and lower abdomen, which was rather distended and tym- panitic; the bladder was felt up to the level of the umbilicus. Both kidneys were easily felt, particularly the right, but did not seem to be enlarged. The penis, testicles and scrotum looked normal. Catheterization withdrew $4 \mathrm{oz}$. of dirty urine and rectal examination revealed an apparently painless, smooth and rounded mass in the pelvis, which prevented full introduction of the examining finger. The mass was situated rather lower down than would be expected for the bladder, although there seemed to be continuity of the two structures, the bladder wall being thickened and hard. The child's temperature was $96 \cdot 4^{\circ} \mathrm{F}$., but had risen to $101 \cdot 4^{\circ} \mathrm{F}$. next morning. The diagnosis of peri-prostatic abscess was made.

Laboratory investigations gave the following results: (25.10.57.) blood urea $79 \mathrm{mg} . / 100 \mathrm{ml}$.; serum electrolytes: Cl $578 \mathrm{mg} . / 100 \mathrm{ml}$. (99 mEq./1.), Na $310 \mathrm{mg} . / 100 \mathrm{ml}$. (134 mEq./1.), K $20 \mathrm{mg} . / 100 \mathrm{ml}$. (5.1 mEq./l.); $\mathrm{CO}_{2} 64.7$ vol. $\%(29.2 \mathrm{mEq} . / 1.) ; \mathrm{Hb} 79 \%$ or 11.60 g. $/ 100 \mathrm{ml}$; W.B.C. 29,500 ; neutrophils $65 \%$; P.C.V. $40 \%$; urine slightly alkaline; protein $80 \mathrm{mg} . \%$; deposits nil; culture (catheter specimen) no growth.

At operation a needle was introduced through the perineum in front of the rectum; it entered the cavity of a peri-prostatic abscess and $20 \mathrm{ml}$. of thick greenish pus were removed with a syringe. Afterwards the opening was enlarged with a sinus forceps and a corrugated rubber drain was left in place. The pus showed a very large number of polymorphs and gram-positive cocci, which on culture produced a heavy growth of coagulasepositive staphylococci.

Following the operation micturition was normal. The
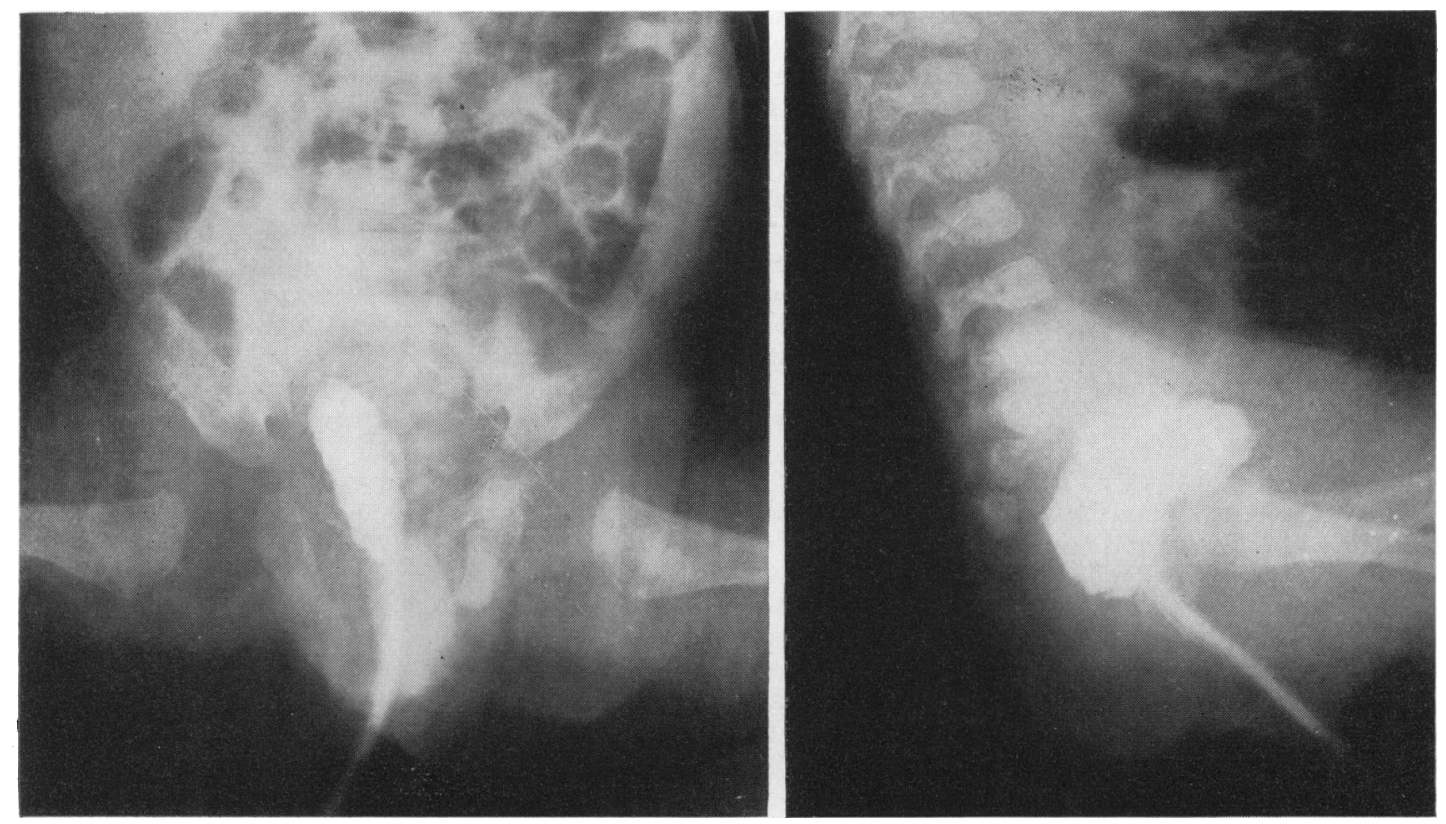

FIG. 1.-Case 2. Sinograms outlining haematoma cavity. 
sinus had to be opened with forceps on two separate occasions, but he then had an uneventful convalescence and was discharged on November 9. The perineal wound was healed, he was taking feeds well, gaining weight, and had a good stream of urine. Rectal examination was normal apart from slight induration of the prostatic region.

Eighteen months later his adoptive parents wrote to say that he was in excellent health, and as they were living a long way from London they were not prepared to bring him up for examination.

Case 4. A third child was born at full term on May 21,1958 , in a normal delivery. The mother's pregnancy and puerperal period were uneventful. He weighed $8 \mathrm{lb} .12 \mathrm{oz}$. at birth.

During the first week his stools were rather frequent, small in amount and brownish in colour, and he lost weight steadily, looking rather lethargic. On June 2 he vomited once before and once after a feed. The vomit was at times projectile, containing some brown material but no definite macroscopical evidence of blood. He was sent to The Hospital for Sick Children with a diagnosis of pyloric stenosis.

On admission under Dr. Wilfrid Sheldon on June 3 he looked rather sallow but his hydration was fair, his weight being $7 \mathrm{lb} .13 \mathrm{oz}$. The abdomen showed no visible peristalsis and no pyloric tumour was felt. The liver was two fingers below the costal margin with a slightly irregular edge. The kidneys were enlarged and lobulated and the blood urea was $208 \mathrm{mg}$.\%. A routine urine analysis showed infection with Esch. coli, and a blood culture performed the same day also showed a heavy growth of Esch. coli. Next morning the spleen was palpable and the child was put on Achromycin.

Two days later there was some generalized oedema, the kidneys were still enlarged and a distended bladder was also palpated. The blood urea was still raised and bilateral pyelostomies were performed for drainage. At operation the kidneys were found to be large but looked healthy and the pelves were not unduly distended. On the following day an examination under anaesthesia revealed a smooth round prostatic swelling. A needle was introduced through the perineum and altered blood was aspirated. An incision was made and a large amount of blood evacuated. A corrugated rubber drain was left in the wound and an indwelling catheter was used for bladder drainage. The blood produced on culture a heavy growth of Esch. coli.

In the afternoon following operation the child was still pale and lethargic and he was given a small blood transfusion. Next day the prostatic swelling had lessened and some more blood clots were expressed with the help of a rectal finger. The nephrostomy tubes were then removed. He was kept on intravenous fluids until June 10 , and by that time his general condition had considerably improved. On June 11, although the kidneys were still palpable and the bladder distended at times, he was able to pass urine with a fair stream.

Subsequently he made only slow progress, interrupted by repeated urinary infections. By July 7 the blood urea had fallen to $41 \mathrm{mg}$. $/ 100 \mathrm{ml}$., but micturition was not satisfactory. An intravenous pyelogram then showed considerable bilateral dilatation of the ureters and later an expression cystogram revealed the presence of typical congenital posterior urethral valves. This obstruction was treated endoscopically and normal micturition was restored but persistent urinary infection remained a troublesome feature and in November the excessively tortuous left ureter was shortened and straightened. Following that operation progress was more satisfactory, though there was still a slight pyuria.

In this case, therefore, the effusion of blood only exacerbated a pre-existing congenital obstruction.

Case 5. A first child was born at full term on May 5, 1953 , in a normal delivery. The mother's pregnancy and puerperal period were uneventful. He weighed $6 \mathrm{lb} .13 \mathrm{oz}$. at birth and thrived on breast feeding supplemented by a bottle. He had no complaints until 2 weeks old when he was noticed to have a slight whitish discharge from beneath the prepuce and seemed to have pain on micturition, straining and screaming during the act. The urine looked cloudy, thick and whitish and the stream was reduced to a continuous dribble. On account of worsening of these symptoms, he was admitted on May 29 to Amersham General Hospital under Dr. Dermod MacCarthy, and the mucoid penile discharge was found to contain numerous pus cells. The abdomen was not distended but the bladder was full. On rectal examination there was a tense elastic swelling bulging anteriorly into the rectum, encountered immediately by the palpating finger. Following admission he passed increasing amounts of urine, with pus present macroscopically. A urine specimen showed very numerous pus cells and on culture grew Staphylococcus pyogenes. He received penicillin 100,000 units twice daily. On May 30, after passing about $1 \mathrm{oz}$. of pink pus, his urine became quite clear and pain on micturition almost disappeared. His temperature was never above $99 \cdot 6^{\circ} \mathrm{F}$., and throughout he took his feeds well, never vomiting. At this stage he was transferred to The Hospital for Sick Children. On admission he looked a normal healthy boy with good colour and hydration and weighed $7 \mathrm{lb} .12 \mathrm{oz}$. No umbilical infection or septic spots were noticed, the bladder was not distended, and the foreskin and urethral meatus were normal, no discharge being seen. Rectal examination showed a normal anus and rectum and a soft but enlarged prostate (more on the right side than on the left). No other relevant findings were detected on clinical examination. Within a week the prostatic swelling completely subsided, leaving only a slight induration. Bowel actions were normal, appetite was good, temperature was normal and the child was gaining weight. Micturition was normal with a good stream of clear urine when the child was discharged on June 6.

Investigations showed: (31.5.53.) blood urea $23 \mathrm{mg} . / 100 \mathrm{ml}$.; Hb $102 \%$ or $14.3 \mathrm{~g} . / 100 \mathrm{ml}$.; R.B.C. $4,290,000$; W.B.C. 23,200; neutrophils $53 \%$; urine neutral, no protein; heavy growth of Esch. coli and moderate growth of coagulase-negative staphylococci on culture. 
Summary

Prostatic abscess and peri-prostatic haematoma are both lesions which cause a severe lower urinary tract obstruction in young infants: they are not necessarily associated with a congenital abnormality and the prognosis is good if the cavity is adequately drained. The abscess is probably metastatic in origin or may follow suppuration in a haematoma, but no satisfactory explanation of the haematoma formation is advanced.

The diagnosis is easily made by rectal palpation after the bladder has been emptied by catheter. A strictly prostatic abscess may be difficult to distinguish from a tumour by palpation but an exploratory needle puncture will settle the issue. The peri-prostatic haematoma is based on the lateral pelvic wall and does not resemble a visceral swelling.

Urinary retention is relieved by drainage of the abscess or haematoma cavity and neither a urethral catheter nor cystostomy is required as long as the cavity is kept empty. Perineal drainage is satisfactory if combined with daily 'stripping' from the rectum. Chemotherapy is required as an adjunct.

\section{REFERENCES}

Alison, F. and Le Tan Vinh (1952). Acute suppurative prostatitis of the newborn. Nourrisson, 40,15.

Boy, J.-L., Wallon, D. and Lauras, H. (1953). Prostatite suppurée à staphylocoque chez un nourrisson de 5 jemaines. Arch. franc. Pédiat. 10,1071.

Campell. ${ }^{2}$. (1929). Gonococcal prostatic abscess in infants. J. Urol. (Baltimore), $22,445$.

Fox, C. P. (1934). Gonorrheal prostatic abscess in four year old boy. J. Amer. med. Ass., 103, 748.

George, P. (1953). Gt. Ormond Str. J., 5, 58.

Szenkier, D. (1929). Prostatic abscess: a case in a boy aged $2 \frac{1}{2}$ years. Z. Uroi., 23, 119. 